\title{
Energy-Efficient MIMO Multihop Communications Using the Antenna Selection Scheme
}

\author{
Fernando Rosas*, Richard Demo Souza ${ }^{\ddagger}$, Marian Verhelst* and Sofie Pollin* \\ * Departement Elektrotechniek, KU Leuven, Belgium \\ $\ddagger$ Electronics Department, UTFPR, Brazil
}

\begin{abstract}
Wireless communications are in general more energy-consuming than wired ones because of the effects of path loss and fading. However, it is well-known that multihop techniques are effective for reducing the path loss cost, while large antenna arrays can increase the diversity gain. Hence, a joint-optimization of the number of hops and the antenna array size would provide a useful scheme for designing energyconstrained wireless applications. In this work we study this joint-optimization in the context of low-power wireless networks, focusing on the antenna selection scheme.

Results show that, regardless of their high power consumption, receive antenna arrays of more than 60 elements are optimal for long-range communications (more than 150 meters) from the point of view of the overall energy budget. In contrast, using a single transmit antenna is the best choice due to the channel estimation cost. Results also show that the energy consumption of optimized systems with large receive antenna arrays can be up to 3.4 times smaller than the consumption of single antenna multihop systems. Therefore, systems with large antenna arrays can be attractive for reducing the traffic in crowded energyconstrained wireless networks, as they can be more energyefficient using less but longer hops than single-antenna systems.
\end{abstract}

\section{INTRODUCTION}

Developing techniques for increasing the energy efficiency of wireless communications is a central requirement for wireless sensor networks (WSN) to prosper into large-scale autonomous systems [1]. Wireless data transfer is the most energy-consuming task that the nodes of these networks perform and, hence, increasing its efficiency has a direct impact on the network lifetime [2]. The costs of wireless communications are specially critical when nodes have to send data over long distances, as in networks that perform surveillance over large areas like roads, industrial parks, battlefields, active volcanoes or natural parks.

In general, wireless communications are more energyconsuming than wired ones mostly because of the effects of large-scale path loss and small-scale fading: the former increases the irradiated power that is required to reach a given destination, and the latter increases the variability of the received signal strength resulting in a larger number of retransmissions due to decoding errors [3]. Multihop techniques can be used to reduce the costs of path loss by using intermediate nodes as relays between sender and destination [1]. Also, multiple-input multiple-output (MIMO) systems can effectively reduce the impact of fading by increasing the diversity gain of the channel [4].

The question that drives this work is how to jointly-optimize the number of hops and the antenna array sizes of the nodes of a MIMO multihop wireless network in order to maximize the number of data bits that can be transferred per energy unit. This manuscript constitutes a first step towards an answer to that question, providing an analysis of the energy consumption of MIMO multihop systems where the MIMO antenna selection scheme is used. Antenna selection allows to considerably reduce the power consumption of the baseband electronic components when compared to other spatial diversity techniques like space time block codes or transmit/receive beamforming [5]. Recently, it has been shown that antenna selection is an attractive solution for energy-constrained single hop cellular systems [6] and single hop cooperative WSN [7].

The idea of combining MIMO and multihop strategies was previously presented in [8], where uncoded single-input single output (SISO) and $2 \times 2 \mathrm{MIMO}$ transmissions were compared. In [9] the energy efficiency of single-input multiple-output (SIMO) multihop links using maximum ratio combining on Rayleigh channels was studied. Recently [10], [11] have addressed the issue of power allocation on MIMO multihop networks. These works do not consider the effect of decoding errors or the costs of the MIMO channel estimation rounds, which are known to be critical issues [12].

As opposed to much of the existing literature, our energymodeling framework is not information theoretical but based on signal models. Following [3], our approach accounts the costs of retransmissions due to decoding errors of concrete modulation and coding schemes. Our work is also, to the best of our knowledge, the first to take into account the overall impact of the costs of overhead and channel estimation periods and the impact of the coding scheme in a MIMO multihop scenario. Finally, our approach is also novel in including simultaneously an arbitrary number of hops and arbitrary large antenna arrays.

The rest of this article is organized as follows: Sections II and III develop Link Layer and Physical Layer aspects of our energy consumption model, Section IV presents numerical results and finally Section V summarizes our conclusions.

\section{ENERGY CONSUMPTION MODEL}

Our goal is to determine the total energy that is necessary for transferring one bit of data successfully in a point-topoint packet-switched MIMO multihop link. Such a bit is called a "goodbit". Following [3], every frame transmitted in the forward direction is matched by a feedback frame in the reverse direction that acknowledges correct reception or 
requests a retransmission. It is also assumed that all frames in both directions are detected and that all feedback frames are decoded without error.

We study the energy consumption of a MIMO multihop link in which the nodes use the antenna selection scheme [13]. In this MIMO scheme the receiver determines the receive and transmit antenna pair with the highest signal-to-noise ratio (SNR), and sends this information back to the transmitter via a feedback channel. After the MIMO channel estimation has been performed, both transmitter and receiver keep only one active RF chain for doing the data transmissions. It is assumed that each node of the multihop path uses the same transmission scheme, and while they have $N_{\text {total }}$ antennas they select the best from subsets of $N_{\mathrm{t}}$ transmit and $N_{\mathrm{r}}$ receive antennas.

In the sequel, Section II-A (resp. Section II-B) presents the analysis of the energy required for transmitting (resp. receiving) a goodbit, and then Section II-C synthesizes the total energy consumption model of a MIMO multihop link.

\section{A. Components of Energy Consumption at the Transmitter}

The energy consumption of the transceiver that transmits forward frames and receives feedback frames is composed of three terms, each one described next.

1) Communication costs: Following [3], the energy consumption per goodbit for transmitting forward frames and receiving feedback frames can be calculated as

$$
\mathcal{E}_{\mathrm{tx}}=\left[\left(P_{\mathrm{el}, \mathrm{tx}}+P_{\mathrm{PA}}\right) T_{\mathrm{b}}+P_{\mathrm{el}, \mathrm{rx}} T_{\mathrm{fb}}\right] \tau_{\mathrm{tx}},
$$

where $P_{\mathrm{PA}}$ is the power consumed by the power amplifier (PA) of the selected RF branch, $P_{\text {el,tx }}$ (respectively $P_{\text {el,rx }}$ ) is the total power consumed by the remaining baseband and radio-frequency electronic components of the selected RF branch that performs the forward transmission (respectively the feedback frame reception) and $T_{\mathrm{b}}$ (respectively $T_{\mathrm{fb}}$ ) is the average air time per payload bit on a forward frame (respectively a feedback frame). Finally, $\tau_{\mathrm{tx}}$ is the number of transmission trials until a frame is decoded without errors in the receiver, whose properties are analyzed in Section III-B.

Let us now define expressions for $T_{\mathrm{b}}$ and $T_{\mathrm{fb}}$. Define $r=k / n$ as the code rate, where $n$ is the number of bits per codeword and $n-k$ is the number of added redundancy bits. Then, each physical-layer forward frame carries $L_{\mathrm{h}}$ bits of header and a payload composed by $r L_{\mathrm{p}}$ bits of data and $(1-r) L_{\mathrm{p}}$ additional bits for coding. The total duration of a forward frame is composed by $T_{\mathrm{p}}$ seconds for transmitting the $L_{\mathrm{p}}$ bits of payload (with a suitable modulation), $T_{\mathrm{h}}$ seconds for the transmission of the header (with a binary modulation) and $T_{\mathrm{o}}$ seconds for the transmission of overhead signals (synchronization, etc). The average air time per data bit in a forward frame is $T_{\mathrm{b}}=\left(T_{\mathrm{p}}+T_{\mathrm{h}}+T_{\mathrm{o}}\right) /\left(r L_{\mathrm{p}}\right)$. Let us define $R_{\mathrm{s}}$ as the physical layer symbol-rate and $b=\log _{2} M$ be the number of bits per symbol in a $M$-QAM modulation. By considering that header bits are sent using a binary modulation, and noting that $L_{\mathrm{p}} / T_{\mathrm{p}}=b R_{\mathrm{s}}$, then one can express $T_{\mathrm{b}}$ as

$$
T_{\mathrm{b}}=\frac{1}{r R_{\mathrm{s}}}\left(\frac{1}{b}+\frac{L_{\mathrm{h}}}{L_{\mathrm{p}}}+\frac{L_{\mathrm{o}}}{L_{\mathrm{p}}}\right),
$$

where $L_{\mathrm{o}}$ is the length of the overhead measured in bits. Analogously, one finds that

$$
T_{\mathrm{fb}}=\frac{L_{\mathrm{f}}}{r b R_{\mathrm{s}} L_{\mathrm{p}}}
$$

is the feedback time per payload bit, where $L_{\mathrm{f}}$ is the number of bits per feedback frame.

2) MIMO channel estimation rounds: For estimating the MIMO channel a pre-defined sequence of symbols is transmitted sequentially by each transmit antenna and received simultaneously by all the receive antennas. This procedure has to be updated every time the wireless channel changes significantly, i.e. at least once per coherence time. Therefore, the energy consumption of each channel estimation round per goodbit can be calculated as

$$
\mathcal{E}_{\mathrm{ch}}^{\mathrm{tx})}=\left(P_{\mathrm{el}, \mathrm{tx}}+P_{\mathrm{PA}}\right) T_{\mathrm{ch}} \tau_{\mathrm{ch}},
$$

where $\tau_{\text {ch }}$ is the number of times a channel estimation round has to be performed per goodbit and $T_{\mathrm{ch}}$ is the time required by the transmission of the channel estimation sequence per data bit, which is calculated as

$$
T_{\mathrm{ch}}=\frac{N_{\mathrm{t}} L_{\mathrm{ch}}}{r R_{\mathrm{s}} L_{\mathrm{p}}},
$$

where $L_{\mathrm{ch}}$ is the number of bits of channel estimation sequence that is sent by each transmit antenna.

3) Encoding costs: Most of the costs of encoding forward frames, which are shared among the $r L_{\mathrm{p}}$ data bits, are due to the computations required for implementing the error correcting code (ECC). This can be calculated as $\mathcal{E}_{\text {enc }}=\mathcal{E}_{\text {op }} n_{\text {enc }}^{\text {tot }}$, where $\mathcal{E}_{\mathrm{op}}$ is the energy consumption per operation of the arithmetic processing unit (APU) and $n_{\text {enc }}^{\text {tot }}$ is a shorthand notation for the total number of operations for encoding per goodbit, which is calculated as

$$
n_{\mathrm{enc}}^{\mathrm{tot}}=\frac{1}{r L_{\mathrm{p}}} \sum_{j=1}^{J_{\mathrm{APU}}} c_{j} n_{j}^{\mathrm{enc}, \mathrm{ECC}}
$$

where $c_{j}$ is the number of operations required by the $j$-th APU instruction (addition, multiplication, etc) which is performed $n_{j}^{\text {enc,ECC }}$ times during the ECC algorithm and $J_{\mathrm{APU}}$ is the number of different instructions the APU performs.

\section{B. Components of Energy Consumption at the Receiver}

Let us now describe the terms which characterize the energy consumption at the receiver. The energy that is required for receiving one correct data bit can be calculated as

$$
\mathcal{E}_{\mathrm{rx}}=\left[P_{\mathrm{el}, \mathrm{rx}} T_{\mathrm{b}}+\left(P_{\mathrm{el}, \mathrm{tx}}+P_{\mathrm{PA}}\right) T_{\mathrm{fb}}\right] \tau_{\mathrm{tx}},
$$

where all the terms are as defined in (1). Following (4), the energy consumption of the MIMO channel estimation round in the receiver is given by

$$
\mathcal{E}_{\mathrm{ch}}^{(\mathrm{rx})}=N_{\mathrm{r}} P_{\mathrm{el}, \mathrm{tx}} T_{\mathrm{ch}} \tau_{\mathrm{ch}},
$$

where the $N_{\mathrm{r}}$ factor in (8) comes from the fact that all the receive antennas are actively working. Finally, the energy consumption of decoding the forward frame can be expressed 
as $\mathcal{E}_{\mathrm{dec}}=\mathcal{E}_{\mathrm{op}} n_{\mathrm{dec}}^{\text {tot }}$, where $n_{\mathrm{dec}}^{\text {tot }}$ is the total number of operations for decoding the frame which is calculated as

$$
n_{\mathrm{dec}}^{\mathrm{tot}}=\frac{\tau_{\mathrm{tx}}}{r L_{\mathrm{p}}} \sum_{j=1}^{J_{\mathrm{APU}}} c_{j} n_{j}^{\mathrm{dec}, \mathrm{ECC}},
$$

where all the terms are as defined in (6). Note that, in contrast to (6), the decoding of the ECC has to be performed once per transmission trial - which causes the extra $\tau_{\text {tx }}$ factor in (9).

\section{Energy Consumption of a Multihop Network}

Let us now assume that the multihop path contains $H$ hops of the same length. Using the notation introduced in Sections II-A and II-B, the first node of the chain consumes $\mathcal{E}_{0}=\mathcal{E}_{\mathrm{tx}}+\mathcal{E}_{\mathrm{ch}, \mathrm{tx}}+\mathcal{E}_{\mathrm{enc}}$, the intermediate nodes consume $\mathcal{E}_{i}=\mathcal{E}_{\mathrm{rx}}+\mathcal{E}_{\mathrm{ch}, \mathrm{rx}}+\mathcal{E}_{\mathrm{dec}}+\mathcal{E}_{\mathrm{tx}}+\mathcal{E}_{\mathrm{ch}, \mathrm{tx}}+\mathcal{E}_{\mathrm{cod}}(i=2, \ldots, H-1)$, while the last node consumes $\mathcal{E}_{H}=\mathcal{E}_{\mathrm{rx}}+\mathcal{E}_{\mathrm{ch}, \mathrm{rx}}+\mathcal{E}_{\mathrm{dec}}$. Hence, the total energy consumption is

$$
\begin{aligned}
\mathcal{E}_{\mathrm{b}}^{\mathrm{MH}} & =\sum_{i=0}^{H} \mathcal{E}_{i} \\
& =H\left[\mathcal{E}_{\mathrm{enc}}+\mathcal{E}_{\mathrm{dec}}+\mathcal{E}_{\mathrm{ch}}^{(\mathrm{tx})}+\mathcal{E}_{\mathrm{ch}}^{(\mathrm{rx})}+\mathcal{E}_{\mathrm{tx}}+\mathcal{E}_{\mathrm{rx}}\right] \\
& =H\left(n_{\mathrm{enc}}^{\mathrm{tot}}+n_{\mathrm{dec}}^{\mathrm{tot}}\right) \mathcal{E}_{\mathrm{op}} \\
& \cdots+H\left(P_{\mathrm{el}, \mathrm{tx}}+P_{\mathrm{PA}}\right)\left(T_{\mathrm{b}} \tau_{\mathrm{tx}}+T_{\mathrm{fb}} \tau_{\mathrm{tx}}+T_{\mathrm{ch}} \tau_{\mathrm{ch}}\right) \\
& \cdots+H P_{\mathrm{el}, \mathrm{rx}}\left(T_{\mathrm{b}} \tau_{\mathrm{tx}}+T_{\mathrm{fb}} \tau_{\mathrm{tx}}+N_{\mathrm{r}} T_{\mathrm{ch}} \tau_{\mathrm{ch}}\right) .
\end{aligned}
$$

Let us introduce the following notation: $n^{\text {tot }}=n_{\mathrm{enc}}^{\text {tot }}+n_{\mathrm{dec}}^{\text {tot }}$ as the total number of operations per goodbit, $T=T_{\mathrm{b}}+T_{\mathrm{fb}}$ as the total transmission time per bit, $T_{\text {ant }}=T_{\mathrm{ch}} / N_{\mathrm{t}}$ for the duration of the channel estimation round per transmit antenna and $\chi=T_{\text {ant }} / T$. Let us further assume than an acquisition round is required for each transmitted frame, and hence $\tau_{\mathrm{ch}}=$ $\tau_{\mathrm{tx}}:=\tau$. Then, the energy consumption per goodbit is

$$
\begin{aligned}
\mathcal{E}_{\mathrm{b}}^{\mathrm{MH}}= & H n^{\text {tot }} \mathcal{E}_{\mathrm{op}}+H T\left(P_{\mathrm{el}, \mathrm{tx}}+P_{\mathrm{PA}}\right)\left(1+N_{\mathrm{t}} \chi\right) \tau \\
& \cdots+H T P_{\mathrm{el}, \mathrm{rx}}\left(1+N_{\mathrm{r}} N_{\mathrm{t}} \chi\right) \tau .
\end{aligned}
$$

\section{ENERGY CONSUMPTION AS FUNCTION OF THE SNR}

The goal of this section is to further develop the energy consumption model ir order to make explicit the role of the SNR. In the following, Section III-A studies the PA power consumption and Section III-B analyzes the retransmission statistics. Finally, Section III-C syntetizes these results with the ones presented in the Section II and presents a qualitative analysis.

\section{A. PA Consumption}

Let us relate the power consumption of the PA with the SNR. The selected transmit antenna radiates on average $\bar{P}_{\text {rf }}$ Watts provided by the corresponding PA. The PA's power consumption is modeled by

$$
P_{\mathrm{PA}}=\frac{1}{\eta} \bar{P}_{\mathrm{rf}}
$$

where $\eta$ the average efficiency of the PA. If we limit the analysis to linear PAs (such as Class A or B PAs), then we can approximate $\eta$ with [14]

$$
\eta=\left(\frac{\bar{P}_{\mathrm{rf}}}{P_{\max }}\right)^{\beta} \eta_{\max },
$$

where $P_{\max }$ is the maximal PA output and $\beta$ and $\eta_{\max }$ are parameters which depend on the PA class. In these equations, $P_{\text {back-off }}=P_{\text {max }} / \bar{P}_{\text {rf }}$ is the back-off of the PA. In general, one can calculate the back-off as $P_{\text {back-off }}=\xi / S$, where $\xi$ is the peak-to-average power ratio of the modulation (which for MQAM modulations is calculated as $\xi=3[\sqrt{M}-1] /[\sqrt{M}+1]$ ) and $S$ accounts for any additional back-off that may be taken. Hence, the relationship between the PA consumption $P_{\mathrm{PA}}$ and the average radiated power $\bar{P}_{\text {rf }}$ is calculated as

$$
P_{\mathrm{PA}}=\frac{\xi^{\beta}}{\eta_{\max } S^{\beta}} \bar{P}_{\mathrm{rf}} \text {. }
$$

The transmission power attenuates over the air with path loss and arrives at the receiver with a mean power given by

$$
\bar{P}_{\mathrm{rx}}=\frac{\bar{P}_{\mathrm{tx}}}{A_{0}(d / H)^{\alpha}},
$$

where $A_{0}$ is a parameter that depends on the transmit and receive antenna gains and the transmission wavelength, $d / H$ is the distance between the original transmitter and final receiver divided by the number of hops and $\alpha$ is the path loss exponent. The total received signal power is $\bar{P}_{\mathrm{rx}}=\sigma_{\mathrm{n}}^{2} \bar{\gamma}$, where $\sigma_{\mathrm{n}}^{2}$ is the thermal noise power and $\bar{\gamma}$ is the average SNR. In general, $\sigma_{\mathrm{n}}^{2}=N_{0} W N_{\mathrm{f}} M_{\mathrm{L}}$, where $N_{0}$ is the power spectral density of the baseband-equivalent additive white Gaussian noise (AWGN), $W$ is the transmission bandwidth, $N_{\mathrm{f}}$ is the noise figure of the receiver's front end and $M_{\mathrm{L}}$ is a link margin term which represents any other additive noise or interference [15]. Combining this with (16) and (17) one finds that

$$
\begin{aligned}
P_{\mathrm{PA}} & =\left(\frac{\xi}{S}\right)^{\beta} \frac{A_{0}(d / H)^{\alpha}}{\eta_{\max }} \bar{P}_{\mathrm{rx}} \\
& =\left[\left(\frac{\xi}{S}\right)^{\beta} \frac{N_{0} W N_{\mathrm{f}} M_{\mathrm{L}} A_{0}}{\eta_{\max }}\right](d / H)^{\alpha} \bar{\gamma} \\
& =A(d / H)^{\alpha} \bar{\gamma},
\end{aligned}
$$

with $A$ defined by the term within square brackets.

\section{B. Retransmission Statistics}

A key contributor to the energy consumption is the need for retransmissions due to frames that are decoded with errors. By assuming that the transmitter is equipped with a deep interleaver which completely decorrelates the channel between any successive symbols, then it can be shown that the average number of trials until a frame is decoded without error is [3]

$$
\bar{\tau}=\frac{1}{1-\bar{P}_{\mathrm{f}}},
$$

where $\bar{P}_{\mathrm{f}}$ is the mean frame error rate. 
Let us now consider the use of error correcting block codes. For $n<L_{\mathrm{p}}$ lets define $n_{\mathrm{c}}=L_{\mathrm{p}} / n\left(n_{\mathrm{c}} \in \mathbb{N}\right)$ as the number of codewords per payload. Then, to decode a frame correctly one needs $L_{\mathrm{h}}$ correct header bits and $n_{\mathrm{c}}$ codewords with at least $(n-t)=\lambda$ correct symbols, where $t$ is the maximum number of bits that the block code is able to correct per codeword. Hence, by taking into account the various permutations, the probability of correct decoding $\bar{P}_{\mathrm{f}}^{*}=1-\bar{P}_{\mathrm{f}}$ can be written as $\bar{P}_{\mathrm{f}}^{*}=\left[1-\bar{P}_{\mathrm{bin}}(\bar{\gamma})\right]^{L_{\mathrm{h}}}\left[\sum_{j=0}^{t}\left(\begin{array}{c}n \\ j\end{array}\right)\left[1-\bar{P}_{\mathrm{b}}(\bar{\gamma})\right]^{n-j} \bar{P}_{\mathrm{b}}(\bar{\gamma})^{j}\right]^{n_{\mathrm{c}}}$,

where the mean bit error rate (BER) of the $M$-ary modulation $\bar{P}_{\mathrm{b}}(\bar{\gamma})$ is used for the payload bits and the binary modulation BER $\bar{P}_{\text {bin }}(\bar{\gamma})$ is used for the header bits.

Finally, let us now calculate the BER of binary and $M$ ary modulations using a $N_{\mathrm{t}} \times N_{\mathrm{r}}$ MIMO Antenna Selection scheme. Let us assume that the SNR of the channel between the $i$-th receiver and the $j$-th transmitter antennas $\gamma_{i, j}$ are independent and identically distributed exponential random variables, which correspond to the SNR of Rayleigh fading channels*. Therefore their p.d.f. is $p_{\bar{\gamma}}\left(\gamma_{i, j}\right)=\bar{\gamma}^{-1} \exp \left(-\gamma_{i, j} / \bar{\gamma}\right)$, where $\bar{\gamma}$ is the mean SNR per antenna pair. Then, the SNR of the Antenna Selection scheme is given by

$$
\gamma=\max _{\substack{i \in\left\{1, \ldots, N_{\mathrm{r}}\right\} \\ j \in\left\{1, \ldots, N_{\mathrm{t}}\right\}}} \gamma_{i, j}
$$

By using the shorthand notation $\hat{N}=N_{\mathrm{t}} N_{\mathrm{r}}$, the p.d.f. of $\gamma$ can be calculated as follows:

$$
\begin{aligned}
p_{\text {sel }}(\gamma) & =\frac{d}{d \gamma}\left[\int_{0}^{\gamma} p_{\bar{\gamma}}(x) \mathrm{d} x\right]^{\hat{N}} \\
& =\frac{\hat{N}}{\bar{\gamma}} e^{-\frac{\gamma}{\bar{\gamma}}} \sum_{k=0}^{\hat{N}-1} C_{k}^{\hat{N}-1}(-1)^{k} e^{-\frac{k \gamma}{\bar{\gamma}}} \\
& =\sum_{k=0}^{\hat{N}-1} \frac{(-1)^{k} \hat{N} !}{(k+1) !(\hat{N}-k-1) !} \frac{(k+1)}{\bar{\gamma}} e^{-\frac{(k+1) \gamma}{\bar{\gamma}}} \\
& =\sum_{k=1}^{\hat{N}}(-1)^{k-1} C_{k}^{\hat{N}} p_{\bar{\gamma}_{k}}(\gamma),
\end{aligned}
$$

where $C_{k}^{n}=n ! /[k !(n-k) !]$ is the binomial coefficient. This proves that the p.d.f. of the SNR of Antenna Selection can be expressed as a linear combination of $N_{\mathrm{r}} N_{\mathrm{t}}$ p.d.f.s of the SNR of single-antenna Rayleigh channels with mean SNR $\bar{\gamma}_{k}=\bar{\gamma} / k$, with $k \in\left\{1, \ldots, N_{\mathrm{t}} N_{\mathrm{r}}\right\}$. Using this result and the linearity of mathematical expecations, the BER of a given modulation using MIMO antenna selection becomes

$$
\bar{P}_{\mathrm{b}}(\bar{\gamma})=\sum_{k=1}^{N_{\mathrm{t}} N_{\mathrm{r}}}(-1)^{k-1} C_{k}^{N_{\mathrm{t}} N_{\mathrm{r}}} \bar{P}_{\mathrm{b}}^{\text {ray }}\left(\frac{\bar{\gamma}}{k}\right) .
$$

* The more general case of Gamma random variables, which correspond to the Nakagami- $m$ channel fading model, will be addressed in a future extended version of this work
Above, $\bar{P}_{\mathrm{b}}^{\text {ray }}$ denotes the exact BER of $M$-QAM modulations under Rayleigh fading channels, which is given by [16]

$$
\begin{aligned}
& \bar{P}_{\mathrm{b}}^{\text {ray }}(\bar{\gamma})=\frac{1}{\sqrt{M} \log _{2} \sqrt{M}} \sum_{k=1}^{\log _{2} \sqrt{M}} \\
& \cdots \times \sum_{i=0}^{\left(1-2^{-k}\right) \sqrt{M}-1} w(i, k, M)\left(1-\sqrt{\frac{1}{1+\phi(i, M)}}\right)
\end{aligned}
$$

where $\phi(i, M)=2(M-1) /\left[3(2 i+1)^{2} \log _{2} M \bar{\gamma}\right]$ and

$$
w(i, k, M)=(-1)^{\left\lfloor\frac{i 2^{k-1}}{\sqrt{M}}\right\rfloor}\left(2^{k-1}-\left\lfloor\frac{i 2^{k-1}}{\sqrt{M}}+\frac{1}{2}\right\rfloor\right) .
$$

\section{Total Consumption}

Using the results of this section, (13) can be re-written as

$$
\begin{aligned}
\overline{\mathcal{E}}_{\mathrm{b}}^{\mathrm{MH}}= & H \bar{n} \mathcal{E}_{\mathrm{op}}+H T\left[P_{\mathrm{el}, \mathrm{tx}}+A(d / H)^{\alpha} \bar{\gamma}\right]\left[\frac{1+N_{\mathrm{t}} \chi}{1-\bar{P}_{\mathrm{f}}(\bar{\gamma})}\right] \\
& \cdots+H T P_{\mathrm{el}, \mathrm{rx}}\left[\frac{1+N_{\mathrm{r}} N_{\mathrm{t}} \chi}{1-\bar{P}_{\mathrm{f}}(\bar{\gamma})}\right],
\end{aligned}
$$

where $\bar{n}$ is the average total number of APU instructions, which is calculated taking the mean value of (6) and (9) as

$$
\bar{n}=n_{\mathrm{enc}}^{\mathrm{tot}}+\frac{n_{\mathrm{dec}}^{\mathrm{tot}}}{1-\bar{P}_{\mathrm{f}}(\bar{\gamma})}
$$

In (31), the two ratios in square brackets measure the joint impact of the retransmissions and the MIMO channel estimation rounds. For short transmission distances the consumption of the PA is negligible, and therefore it is advantageous to use a large SNR for which $\bar{P}_{\mathrm{f}} \approx 0$. Assuming for simplicity that $P_{\mathrm{el}, \mathrm{tx}} \approx P_{\mathrm{el}, \mathrm{rx}}:=P_{\mathrm{el}}$, then we can write

$$
\overline{\mathcal{E}}_{\mathrm{b}}^{\mathrm{MH}} \approx H \bar{n} \mathcal{E}_{\mathrm{op}}+H T P_{\mathrm{el}}\left[2+N_{\mathrm{t}}\left(1+N_{\mathrm{r}}\right) \chi\right],
$$

which shows that is better to use few hops and few antennas. In contrast, when the total distance $d$ is large, then the PA consumption dominates over the electronic consumption. For those cases, we can write

$$
\overline{\mathcal{E}}_{\mathrm{b}}^{\mathrm{MH}} \approx H^{1-\alpha} T A d^{\alpha} \bar{\gamma}\left[\frac{1+N_{\mathrm{t}} \chi}{1-\bar{P}_{\mathrm{f}}(\bar{\gamma})}\right]
$$

and hence it makes sense to use many hops (as $\alpha \geq 2$ ) and receive antennas, as the latter reduces the value of $\bar{P}_{\mathrm{f}}$ almost for free. Extra transmit antennas provide an equivalent benefit over the error rates while requiring longer channel estimation.

\section{NUMERICAL RESULTS}

This section presents a quantitative analysis of our energy consumption model based on numerical evaluations of (31), (22) and (28). The evaluations were performed using parameters of state-of-the-art low-power devices, which are shown in Table I. We considered Rayleigh fading statistics, Class B PAs and $\mathrm{BCH}$ block codes with variable code rate; the consumption of the latter has been calculated following [17]. For simplicity we considered one codeword per frame (i.e. $n=L_{\mathrm{p}}$ ).

By minimizing (31) the optimal number of hops, SNR, $M$ QAM constellation size (from BPSK up to 64-QAM) and 
TABLE I

GENERIC LOW-POWER DEVICE PARAMETERS

\begin{tabular}{|c|c|c|}
\hline Parameter & Description & Value \\
\hline$L_{\mathrm{p}}$ & Payload size & 1023 bites $\S$ \\
\hline$L_{\mathrm{h}}$ & Frame Header & 5 bytes $\S$ \\
\hline$L_{\mathrm{o}}$ & Overhead & 2 bytes $\S$ \\
\hline$L_{\mathrm{ch}}$ & Channel estimation sequence length & 1 byte per tx antenna \\
\hline$L_{\mathrm{f}}$ & Feedback frame length & 5 bytes $\S$ \\
\hline$W$ & Bandwidth & $1 \mathrm{MHz} \S$ \\
\hline$R_{\mathrm{S}}$ & Symbol rate & $125 \mathrm{KBaud}^{\S}$ \\
\hline$\alpha$ & Path-loss coefficient & 3.2 \\
\hline$A_{0}$ & Free space path loss & $14.92 \mathrm{~dB}$ \\
\hline$\eta_{\max }$ & Maximum PA efficiency & $0.785 \ddagger$ \\
\hline$\beta$ & PA exponent & $0.5 \ddagger$ \\
\hline$S$ & Additional back-off coefficient & $0 \mathrm{~dB}$ \\
\hline$P_{\mathrm{el}, \mathrm{tx}}$ & Tx electronic power consumption & $11.2 \mathrm{~mW}^{\dagger}$ \\
\hline$P_{\mathrm{el}, \mathrm{rx}}$ & Rx electronic power consumption & $16.6 \mathrm{~mW}^{\dagger}$ \\
\hline$N_{0}$ & Noise power density & $-174 \mathrm{dBm} / \mathrm{Hz}$ \\
\hline$N_{\mathrm{f}}$ & Receiver noise figure & $4.4 \mathrm{~dB}^{\dagger}$ \\
\hline$M_{1}$ & Link margin & $20 \mathrm{~dB}$ \\
\hline $\mathcal{E}_{\mathrm{op}}$ & Energy per APU operation & $100 \mathrm{pJ}^{* *}$ \\
\hline$c_{\mathrm{add}}$ & Addition cost & 1 operation * \\
\hline$c_{\text {prod }}$ & Multiplication cost & 1 operation * \\
\hline$n_{\text {add }}^{\text {decCCC }}$ & Number of sums - ECC & $(2 n-1) t+2 t^{2} *$ \\
\hline$n_{\text {prod }}^{\text {dec,ECC }}$ & Number of products - ECC & $2 n t+2 t^{2} *$ \\
\hline
\end{tabular}

*source: [17], ${ }^{\ddagger}$ source: [14], ${ }^{\S}$ source: [18], ${ }^{\dagger}$ source: [19], ${ }^{* *}$ source: [20]

code rate was determined for various antenna array sizes $\left(N_{\mathrm{t}}, N_{\mathrm{r}} \leq 100\right.$ in our evaluations) and total transmit distances $(d \leq 500 \mathrm{~m})$. For optimizing the SNR, we considered an upper irradiation power limit of $10 \mathrm{dBm}$, which is a typical regulatory constraint value. For the code rate, one selects the optimal among all the available $\mathrm{BCH}$ codes with $n=1023$ [17]. Finally, the energy consumption of different antenna array sizes while using their optimal SNR, $M$-QAM modulation and code rate was evaluated.

In agreement with the analysis presented in Section III-C, numerical results show that SIMO systems (i.e. $N_{t}=1$ ) are optimal for almost all transmission distances: small receive antenna arrays are optimal for short link transmissions, while large ones are good for long range communications (see Figure 1). While the energy consumption of single-hop transmissions grows exponentially with the transmission distance, the consumption tends to grow linearly when using multiple hops (see Figure 2). This tendency holds to any antenna array size, changing only the slope and the intercept. Large SIMO systems have smaller slope and larger intercept than small ones, which turns them into good choices for long range transmissions.

We also found that SISO multihop systems can consume up to 3.4 times more than MIMO multihop systems in long range transmissions (see Figure 3). Remarkably, MIMO multihop systems with large receive antenna arrays can consume less energy while using longer hops than SISO systems (see Figure 4). Therefore, by transferring the data using less transmissions these systems could efficiently help to reduce the congestion in energy-constrained wireless networks.

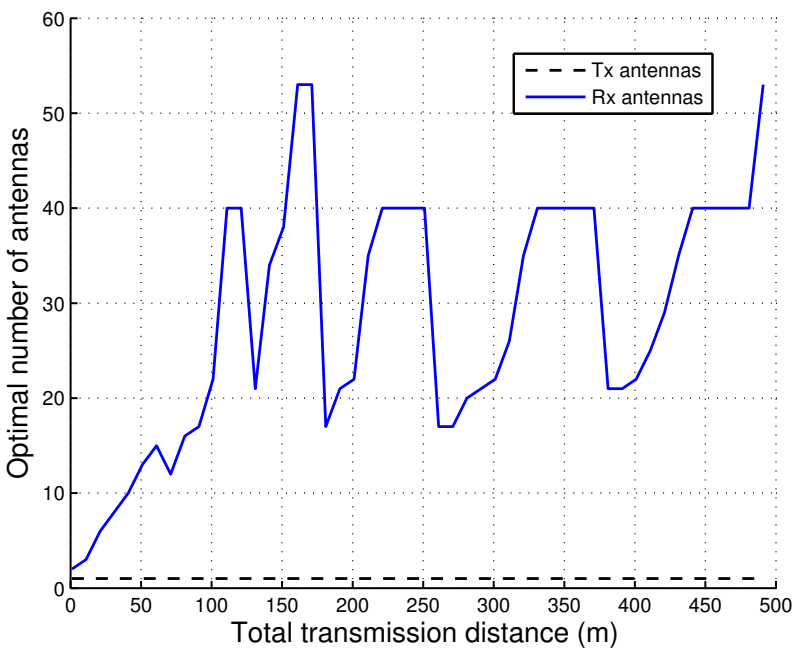

Fig. 1. Optimal transmitter and receiver antenna array sizes for a MIMO multihop link which uses the Antenna Selection scheme.

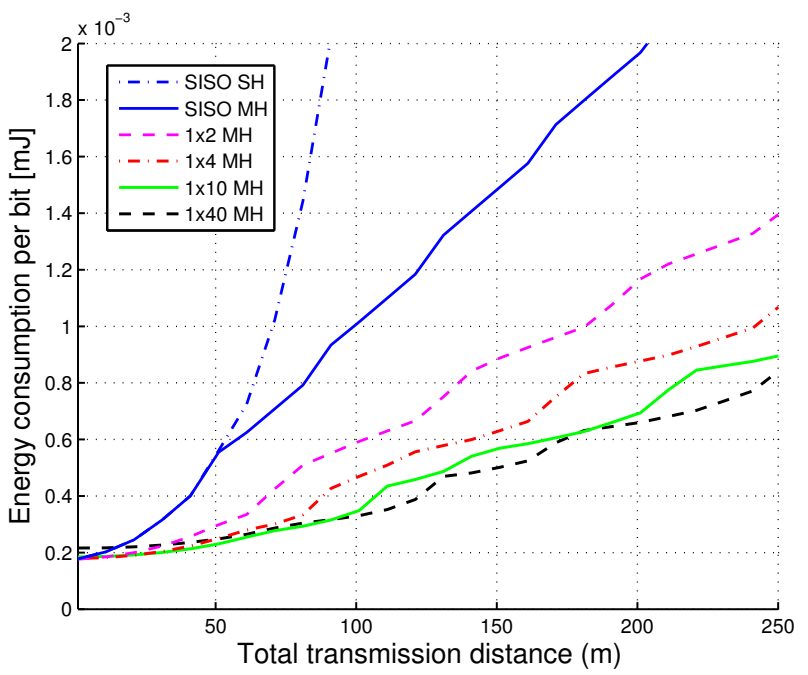

Fig. 2. Performances of SISO singlehop (SH), SISO multihop (MH) and various MIMO multihop links calculated using (31). Each system is evaluated while using its own optimal number of hops, SNR, $M$-QAM constellation size and code rate.

Results also show that the optimal constellation size and the optimal code rate tend to grow as the transmition distance shortens. A detailed analysis of these results will be presented in a future extension of this work due to the lack of space.

Moreover, although one could argue that a WSN node could not handle a large antenna array because of size and costs restrictions, we believe that this could change in the near future [21]. Nevertheless, results also show that most of the energy savings can still be achieved by using only 2 or 4 receive antennas, as the performance of SIMO systems has diminishing returns (see Figure 3).

\section{COnClusions}

Results show that, due to the costs of the MIMO channel estimation rounds, SIMO systems are the optimal choice: sin- 


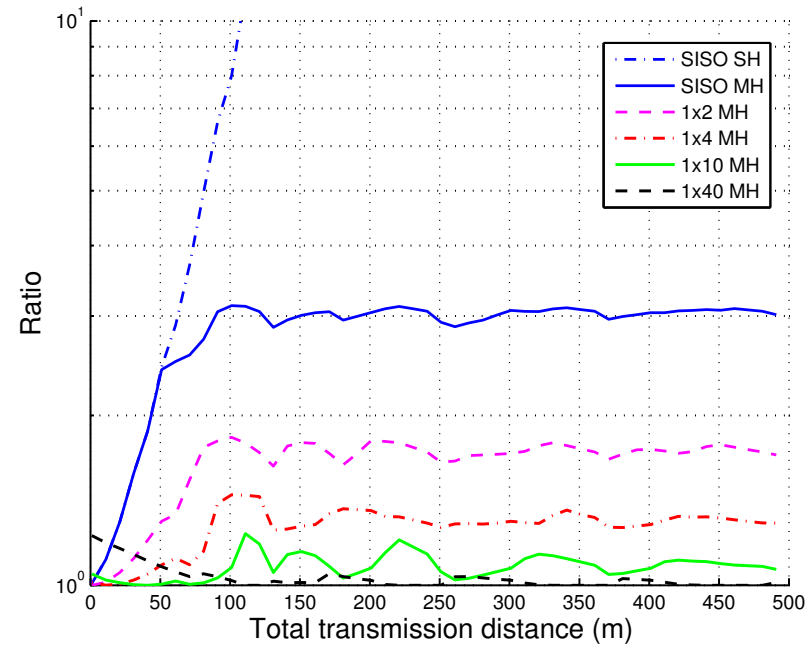

Fig. 3. Ratio of the consumption of various antenna arrays with respect to the consumption of the optimal antenna array size for each total transmit distance.

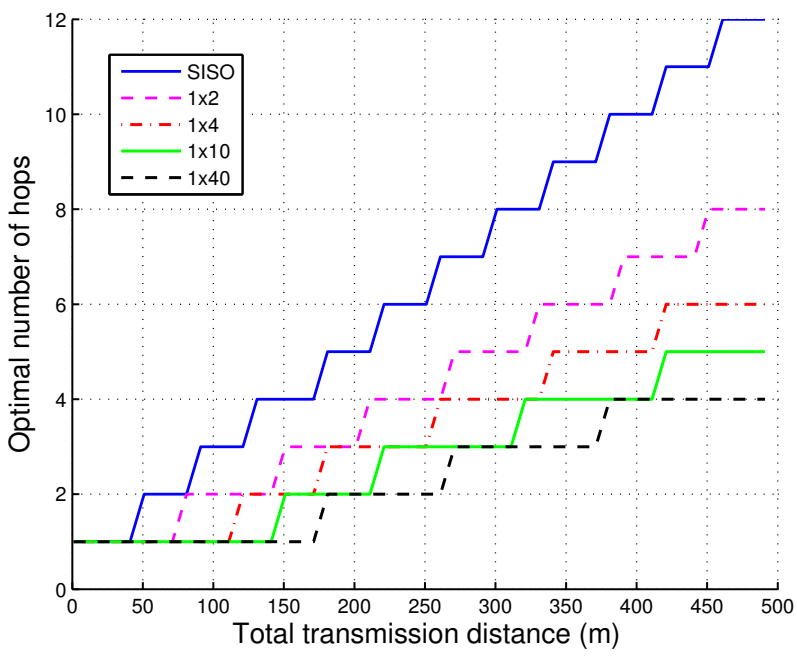

Fig. 4. Optimal number of hops for various antenna array sizes. Large receive antenna arrays can be more efficient than SISO systems while requiring substantially less hops for reaching a given destination.

gle hop transmissions using devices with small antenna arrays when the transmission distance is short, and multihop with large receive antenna arrays for long-range communications. This means that, for long multihop paths, the reduction of the number of hops and irradiated power that large antenna arrays allow compensate the higher power consumption introduced by the more complex electronics that they require.

Results also show that the energy consumption of transmissions using large receive antenna arrays can be up to 3.4 times smaller than using SISO devices. Therefore, large antenna arrays might provide an attractive solution for reducing the traffic in crowded energy-constrained multihop networks, as they can consume less energy while using fewer but longer hops than SISO systems.

\section{ACKNOWLEDGMENTS}

The authors would like to acknowledge the SBO project "SINS", funded by the Agency for Innovation by Science and Technology IWT (Belgium), and the partial support from CAPES and CNPq (Brazil).

\section{REFERENCES}

[1] A. W. Holger Karl, Protocols and Architectures for Wireless Sensor Networks. John Wiley \& Sons inc., 2005.

[2] R. Verdone, D. Dardari, G. Mazzini, and A. Conti, Wireless Sensor and Actuator Network: technologies, analysis and design. Academic Press, 2008.

[3] F. Rosas and C. Oberli, "Modulation and SNR optimization for achieving energy-efficient communications over short-range fading channels," IEEE Transactions on Wireless Communications, vol. 11, no. 12, pp. 4286-4295, 2012.

[4] D. Tse and P. Viswanath, Fundamentals of Wireless Communication. Cambridge University Press, 2005.

[5] S. Sanayei and A. Nosratinia, "Antenna selection in MIMO systems," IEEE Communications Magazine, vol. 42, no. 10, pp. 68-73, 2004.

[6] M. Kakitani, G. Brante, R. Souza, and M. Imran, "Energy efficiency of transmit diversity systems under a realistic power consumption model," IEEE Communications Letters, vol. 17, no. 1, pp. 119-122, 2013.

[7] G. Brante, I. Stupia, R. D. Souza, and L. Vandendorpe, "Outage Probability and Energy Efficiency of Cooperative MIMO with Antenna Selection," IEEE Transactions on Wireless Communications, vol. 12, no. 11, pp. 5896-5907, November 2013.

[8] G. N. Bravos and A. G. Kanatas, "Combining MIMO and MultihopBased Transmissions on Energy Efficient Sensor Networks," Procedings of the Program for European Wireless, 2007.

[9] C.-L. Wang, Y.-W. Huang, and Y.-C. Huang, "An energy-efficient cooperative SIMO transmission scheme for wireless sensor networks," in IEEE ICC'09, 2009, pp. 1-5.

[10] K. Lahetkangas, M. Codreanu, and B. Aazhang, "Energy efficient power allocation for MIMO multihop networks," in IEEE ISIT, 2013, pp. 30353039.

[11] A. Zappone, P. Cao, and E. Jorswieck, "Energy Efficiency Optimization in Relay-Assisted MIMO Systems with Perfect and Statistical CSI," IEEE Transactions on Signal Processing, vol. 62, no. 2, pp. 443-457, Jan 2014.

[12] G. Li, Z. Xu, C. Xiong, C. Yang, S. Zhang, Y. Chen, and S. Xu, "Energyefficient wireless communications: tutorial, survey, and open issues," IEEE Wireless Communications, vol. 18, no. 6, pp. 28-35, Dec. 2011.

[13] A. Goldsmith, Wireless Communications. Cambridge University Press, 2005.

[14] A. He, S. Srikanteswara, K. K. Bae, T. Newman, J. Reed, W. Tranter, M. Sajadieh, and M. Verhelst, "System power consumption minimization for multichannel communications using cognitive radio," in IEEE COMCAS, Nov 2009, pp. 1-5.

[15] S. Cui, A. J. Goldsmith, and A. Bahai, "Energy-constrained modulation optimization," IEEE Transactions on Wireless Communications, vol. 4 no. 5, pp. 2349-2360, 2005.

[16] W. Lopes, W. Queiroz, F. Madeiro, and M. Alencar, "Exact bit error probability of M-QAM modulation over flat rayleigh fading channels," in SBMO/IEEE IMOC, Oct 2007, pp. 804-806.

[17] F. Rosas, G. Brante, R. Souza, and C. Oberli, "Optimizing the code rate for achieving energy-efficient wireless communications," in Proc. WCNC'14, April 2014, pp. 787-792.

[18] Specifications for Local and Metropolitan Area Networks- Specific Requirements Part 15.4, IEEE Std. 802.15.4, 2006.

[19] L. Zhang et al., "A Reconfigurable Sliding-IF Transceiver for 400 MHz/2.4 GHz IEEE 802.15.6/ZigBee WBAN Hubs With Only 21\% Tuning Range VCO," IEEE Journal of Solid-State Circuits, vol. 48, no. 11, pp. 2705-2716, Nov 2013.

[20] Freescale Semiconductor. Freescale Embedded Solutions Based on ARM Technology. [Online]. Available: www.freescale.com/ARMguide

[21] P. Vainikainen et al., "More than 20 antenna elements in future mobile phones, threat or opportunity?" in 3rd European Conference on Antennas and Propagation, March 2009, pp. 2940-2943. 